DOI: $12737 / 25219$

УДК 332.1

МЕТОДИКА МОДЕЛИРОВАНИЯ ПЛАНОВЫХ И ФАКТИЧЕСКИХ ЗНАЧЕНИЙ СТРАТЕГИЧЕСКИХ ПОКАЗАТЕЛЕЙ СОЦИАЛЬНО-ЭКОНОМИЧЕСКОГО РАЗВИТИЯ ВОРОНЕЖСКОЙ ОБЛАСТИ НА ПРИМЕРЕ ПОКАЗАТЕЛЯ «ПРОИЗВОДИТЕЛЬНОСТЬ ТРУДА В ПРОМЫШЛЕННОСТИ» кандидат физико-математических наук, доцент В. В. Сухорукова ${ }^{1}$ доктор экономических наук, профессор Е. А. Яковлева ${ }^{2}$ кандидат экономических наук Н. А. Азарова ${ }^{2}$

1 - Департамент экономического развития Воронежской области, г. Воронеж, Российская Федерация,

2 - ФГБОУ ВО «Воронежский государственный лесотехнический университет имени Г.Ф. Морозова», г. Воронеж, Российская Федерация

В статье рассматривается разработанная авторами методика моделирования достижения плановых и целевых значений индикаторов путем проведения мониторинга процесса реализации «Стратегии социальноэкономического развития Воронежской области», включая выявление рисков их недостижения к 2018 году и к 2020 году. Авторская методика отражает моделирование скорости достижения целевых показателей социальноэкономического развития региона. В каждом конкретном периоде такая методика показывает соотношение запланированной и фактической скоростей. Фактическая скорость может оказаться недостаточной для достижения поставленной цели, что требует поиска новых организационных и ресурсных решений. Фактическая скорость может оказаться выше запроектированной, что требует изучения и оценки оснований такого опережения, чтобы использовать его в интересах ускорения социально-экономического развития Воронежской области. Методика моделирования является одним из ключевых инструментов преодоления «стратегического разрыва» между целями и практикой территориального управления. Его результаты востребованы в обоснованной коррекции значений и состава показателей стратегических целей, выборе (обновлении) мер их достижения. В статье предлагается осуществлять мониторинг плановых и фактических значений целевых индикаторов в несколько этапов, включающий расчет уровня достижения плановых значений целевых индикаторов в отчетном периоде; выявление рисков недостижения целевых значений индикаторов к 2018 году; выявление рисков недостижения целевых значений индикаторов к 2020 году; анализ внутренних и внешних рисков недостижения целевых значений индикаторов к 2018 году; анализ внутренних и внешних рисков недостижения целевых значений индикаторов к 2020 году. Предложенная методика будет способствовать более точному анализу производительности труда в регионе.

Ключевые слова: методика, моделирование, производительность труда, промышленность, стратегия.

\title{
MODELING OF PLANNED AND ACTUAL VALUES OF STRATEGIC INDICATORS OF SOCIAL AND ECONOMIC DEVELOPMENT OF THE VORONEZH REGION ON THE EXAMPLE OF THE INDICATOR «LABOUR PRODUCTIVITY IN INDUSTRY»
}

$\mathrm{PhD}$ in Physics and Mathematics, Associate Professor V. V. Sukhorukova ${ }^{1}$

DSc in Economics, Professor E. A. Yakovleva ${ }^{2}$

$\mathrm{PhD}$ in Economics N. A. Azarova ${ }^{2}$

1 - Economic development Department of Voronezh region, Voronezh, Russian Federation,

2 - Federal State Budget Education Institution of Higher Education «Voronezh State University of Forestry and Technologies named after G.F. Morozov», Voronezh, Russian Federation

Abstract
In the article the authors developed a modeling technique to achieve the planned and target values of indicators by monitoring the process of implementation of the "strategy of social and economic development of the Voronezh region", including identifying risks to their achievement by 2018, and 2020. The author's technique re- 


\section{Управление. Моделирование. Информатика}

flects the modeling of the rate of achievement of target indicators of social and economic development of the region. In each period, this method shows the ratio of planned and actual speeds. The actual speed may not be sufficient to achieve the goal that requires new organizational and resource decisions. The actual speed may be higher than projected, which requires examination and evaluation of the grounds for such advance to use it to accelerate social and economic development of the Voronezh region. The modeling technique is one of the key tools to bridge the "strategic gap" between the goals and practice of territorial administration. Its results are claimed in valid correction of values and strategic goals, choice (upgrading) measures to achieve them. The article proposes to monitor planned and actual values of target indicators in several stages, including calculation of the level of achievement of planned values of target indicators in the reporting period; identification of risks of not achieving target values of indicators by 2018; identifying risks of not achieving target values of indicators by 2020; analysis of internal and external risks of not achieving target values of indicators by 2018; analysis of internal and external risks of not achieving target values of indicators by 2020. The proposed method will contribute to more accurate analysis of labour productivity in the region.

Keywords: methodology, modeling, labour productivity, industry, strategy.

\section{Введение}

Методика моделирования достижения плановых и целевых значений индикаторов путем проведения мониторинга процесса реализации «Стратегии социально-экономического развития Воронежской области», включая выявление рисков их недостижения к 2018-2020 гг. Предлагаемая методика показывает соотношение запланированной и фактической скоростей достижения целевых показателей.

\section{Методика исследования}

Мониторинг является одним из ключевых инструментов преодоления «стратегического разрыва» между целями и практикой территориального управления. Его результаты востребованы в обоснованной коррекции значений стратегических целей, выборе (обновлении) мер их достижения. Мониторинг призван оценить скорость достижения целевых показателей социально-экономического развития региона.

В каждом конкретном периоде такая оценка показывает соотношение запланированной и фактической скоростей. Фактическая скорость может оказаться недостаточной для достижения поставленной цели, что требует поиска новых организационных и ресурсных решений.

Фактическая скорость может оказаться выше запроектированной, что требует изучения и оценки оснований такого опережения, чтобы использовать его в интересах ускорения социально-экономического развития Воронежской области. Состав задач мониторинга включает:

- выявление соответствия плановых и фактических значений показателей целевой функции Стратегии социально-экономического развития Воронежской области;

- идентификацию и анализ причин, определяющих динамику социально-экономического развития Воронежской области;

- определение новых возможностей и угроз развитию Воронежской области;

- трансформацию полученной информации в конкретные задачи стратегического, среднесрочного, оперативного управления, определяющие принятие новых организационных и ресурсных решений. Мониторинг плановых и фактических значений целевых индикаторов Стратегии проводится в несколько этапов:

1) расчет уровня достижения плановых значений целевых индикаторов Стратегии в отчетном периоде;

2) выявление рисков недостижения целевых значений индикаторов к 2018 году;

3) выявление рисков недостижения целевых значений индикаторов к 2020 году;

4) анализ внутренних и внешних рисков недостижения целевых значений индикаторов к 2018 году;

5) анализ внутренних и внешних рисков недостижения целевых значений индикаторов к 2020 году.

Рассмотрим каждый этап подробно. 
1. Расчет уровня достижения плановых значений челевых индикаторов Стратегии в отчетном периоде.

Мониторинг плановых и фактических значений данного целевого индикатора Стратегии в отчетном периоде состоит в оценке достижения запланированных значений целевых индикаторов.

Расчет уровня достижения плановых значений целевых индикаторов Стратегии в отчетном периоде.

Мониторинг плановых и фактических значений целевых индикаторов Стратегии в отчетном периоде состоит в оценке достижения запланированных значений целевых индикаторов.

Уровень отклонения фактического значения индикатора от планового значения определяется:

a) в отношении целевых индикаторов, большее значение которых отражает лучший результат, по формуле 1:

$$
\Delta I_{\Pi}=\left(\frac{\Pi_{2015}}{\Pi_{\Pi, 2015}}-1\right) \times 100,
$$

где $\Pi_{2015}$ - фактическое значение целевого индикатора в отчетном периоде (2015 год);

$\Pi_{\Pi, 2015}$ - прогнозное значение целевого индикатора в отчетном периоде (2015 год);

б) в отношении целевых индикаторов, меньшее значение которых отражает лучший результат, по формуле 2:

$$
\Delta I_{\Pi}=\left(\frac{\Pi_{\Pi, 2015}}{\Pi_{2015}}-1\right) \times 100 .
$$

В каждом периоде такая оценка показывает соотношение запроектированной и фактической скоростей движения (рис. 1).

Условные обозначения:

А - стратегическая цель (показатель)

$\mathrm{t}_{0}$ - базовый период (год)

$\mathrm{t}_{\mathrm{n}}$ - стратегический период (год)

$\mathrm{a}_{\mathrm{t}}, \mathrm{a}_{1}, \mathrm{a}_{2}-$ уровень показателя в отчетном периоде $\mathrm{t}_{1}-$ по плану $\left(\mathrm{a}_{\mathrm{t}}\right)$ или достигнутый $\left(\mathrm{a}_{1}\right.$ и $\mathrm{a}_{2}$ ).

Если уровень отклонения фактического значения индикатора от планового значения по-

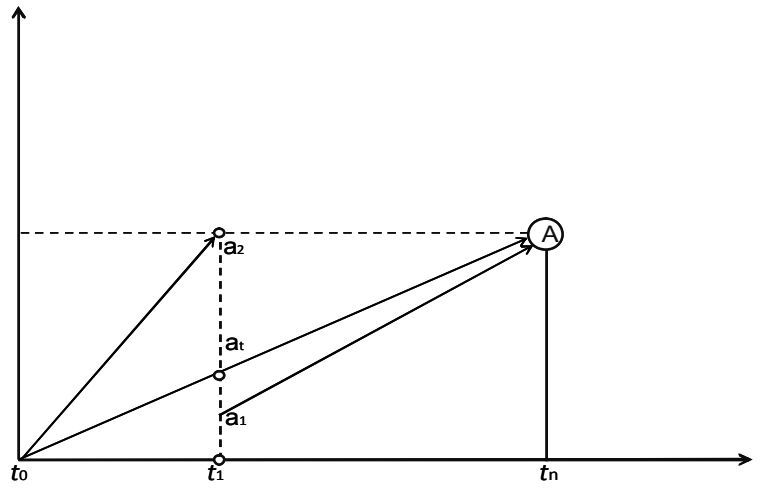

Рис. 1. Оценка скорости движения показателя

ложителен $\left(\Delta \mathrm{I}_{\mathrm{I}}>0\right)$, риск недостижения целевого значения стратегического индикатора отсутствует, при сохранении динамики индикатора. В показанных на рис. 1 примерах эта ситуация соответствует движению к поставленной цели по вектору $\mathrm{t}_{0} \mathrm{a}_{2}$, в этом случае необходимо оценить основания опережения, чтобы использовать их в интересах ускорения развития системы.

Если уровень отклонения фактического значения индикатора от планового значения отрицателен $\left(\Delta \mathrm{I}_{\Pi}<0\right)$, существует риск недостижения стратегических целей, возникает угроза реализации стратегического плана.

Так, в показанных на рисунке примерах со скоростью последующего движения по вектору $\mathrm{a}_{1} \mathrm{~A}$, при котором цель может оказаться недостижимой (трудно достижимой), возникает угроза реализации стратегического плана.

В этом случае необходимо выявить внутренние и внешние причины недостижения плановых значений индикатора, определить пути и механизмы улучшения динамики целевого индикатора.

Большее значение целевого индикатора отражает лучший результат, поэтому уровень отклонения фактического значения индикатора от планового значения определяется по формуле 1 методики:

$$
\Delta I_{\Pi}=\left(\frac{2,1}{2,29}-1\right) \times 100=-8,3 \% .
$$

Уровень отклонения фактического значения индикатора от планового значения отрицателен $\left(\Delta \mathrm{I}_{\Pi}=-8,3 \%<0\right)$, существует риск не- 


\section{Управление. Моделирование. Информатика}

достижения стратегических целей, возникает угроза реализации стратегического плана, цель может оказаться недостижимой (трудно достижимой).

Необходимо выявить внутренние и внешние причины недостижения плановых значений индикатора, определить пути и механизмы улучшения динамики целевого индикатора.

2. Выявление рисков недостижения целевых значений индикатора к 2018 году. Для выявления рисков недостижения целевых значений индикаторов к 2018 году в отчетном периоде необходимо оценить соотношение запроектированной и фактической скоростей движения.

Скорость движения к стратегической цели (на рисунке 1 она задана вектором $\mathrm{t}_{0}-\mathrm{A}$ ) задает целевое значение индикатора в 2018 году. Оно определяется по формуле 3:

$$
\Pi_{L, 2018}=\frac{\Pi_{2020}-\Pi_{2015}}{5} \times 3+\Pi_{2015}=\frac{5,24-2,1}{5} \times 3+2,1=3,98 .
$$

Прогнозное значение целевого индикатора в 2018 году определяется исходя из фактической скорости движения к стратегической цели (среднего темпа роста индикатора) по формуле 4:

$$
\Pi_{\Pi, 2018}=\frac{\Pi_{2015}-\Pi_{2013}}{2} \times 5+\Pi_{2013}=\frac{2,1-1,31}{2} \times 5+1,31=3,29 .
$$

Если полученное значение выражения неположительно, то применяется формула 4а:

$$
\Pi_{\Pi, 2018}=\frac{\Pi_{2015}-\Pi_{2013}}{2} \times 5+\Pi_{2013} .
$$

В сравнении целевого значения индикатора с его фактическим значением определяется уровень отклонения индикатора от поставленной цели в 2018 году:

a) в отношении целевых индикаторов, большее значение которых отражает лучший результат, по формуле 5:

$$
\Delta I_{2018}=\left(\frac{\Pi_{\Pi, 2018}}{\Pi_{L, 2018}}-1\right) \times 100=\left(\frac{3,29}{3,98}-1\right) \times 100=-17,34 \% . .
$$

б) в отношении целевых индикаторов, меньшее значение которых отражает лучший результат, по формуле 6:

$$
\Delta I_{2018}=\left(\frac{\Pi_{Ц, 2018}}{\Pi_{\Pi, 2018}}-1\right) \times 100 .
$$

В случае если уровень отклонения инди- катора от поставленной цели положителен $\left(\Delta \mathrm{I}_{2018}>0\right)$, скорость движения к стратегической цели данного целевого индикатора достаточна для достижения целевого значения индикатора.

В случае если уровень отклонения индикатора от поставленной цели отрицателен $\left(\Delta \mathrm{I}_{2018}\right.$ $<0)$, существует риск недостижения стратегических целей, возникает угроза реализации стратегического плана.

Уровень отклонения индикатора от поставленной цели отрицателен $\left(\Delta \mathrm{I}_{2018}=-17,34 \%\right.$, $\left.\Delta \mathrm{I}_{2018}<0\right)$, существует значительный риск недостижения стратегических целей, возникает угроза реализации стратегического плана. В соответствии с Приложением № 1 к Плану мероприятий по реализации Стратегии социальноэкономического развития Воронежской области на период до 2020 года плановое значение индикатора в 2018 году составляет 3,21 (целевое значение индикатора в 2018 году, задаваемое скорость движения к стратегической цели 2020го года, составляет 3,98). Движение к цели со скоростью ниже средней в 2018 году предполагает наращивание темпов роста показателя в период с 2018 по 2020 год для достижения целевого значения. При планировании более низких темпов роста показателя уровень отклонения индикатора от поставленной цели в 2018 году по формуле 5 методики составит:

$$
\Delta I_{2018}=\left(\frac{\Pi_{n, 2018}}{\Pi_{u, 2018}}-1\right) \times 100=\left(\frac{3,29}{3,21}-1\right) \times 100=2,49 \% .
$$

3. Выявление рисков недостижения целевых значений индикатора к 2020 году.

В случае если существует риск недостижения стратегических целей к 2018 году $\left(\Delta \mathrm{I}_{2018}\right.$ $<0)$, этот риск сохранится и к 2020 году. Оценка уровня недостижения целевых значений к 2020 году проводится по формуле (7):

$$
\Delta I_{2020}=\Delta I_{2018} \times 2=-17,34 \times 2=-34,68 \%
$$

Уровень отклонения индикатора от поставленной цели к 2020 году также отрицателен, при сохранении динамики целевого индикатора риск недостижения стратегических целей к 2020 году будет возрастать.

Анализ внутренних и внешних рисков недостижения целевых значений индикаторов к 
2018 году. В случае если существует риск недостижения стратегических целей, возникает угроза реализации стратегического плана $\left(\Delta \mathrm{I}_{2018}<0\right)$, проводится анализ динамики целевых индикаторов в сравнении со значениями для РФ. Для этого помимо динамики значений целевого индикатора Стратегии за все периоды, начиная с 2005 года и заканчивая отчетным периодом, проводится оценка значений соответствующих индикаторов и показателей макроэкономического развития для РФ за аналогичный период времени [5].

Связь между значениями индикаторов по региону и по РФ определяется с помощью ко-

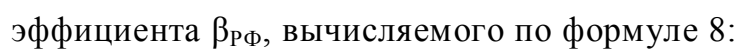

$$
\beta_{P \Phi}=\frac{\operatorname{cov}\left(\Pi_{B O}, \Pi_{P \Phi}\right)}{\sigma_{P \Phi}^{2}},
$$

где

$$
\operatorname{cov}\left(\Pi_{B O}, \Pi_{P \Phi}\right)=\frac{\sum_{k=0}^{i}\left(\Pi_{k}-\Pi_{P \Phi, k}\right)^{2}}{n-1} .
$$

ковариация между значениями целевого индикатора в регионе и по РФ в целом;

$$
\sigma_{P \Phi}^{2}=\frac{\sum_{k=0}^{i}\left(\Pi_{P \Phi, k}-\bar{\Pi}_{P \Phi}\right)^{2}}{n-1} .- \text { стандартное от- }
$$

клонение целевого индикатора от среднего по РФ в целом;

$\Pi_{\mathrm{k}}$ - фактическое значение целевого индикатора в k-ом периоде в регионе;

$\Pi_{\text {РФ,k }}$ - фактическое значение целевого индикатора в k-ом периоде по РФ; k = 2013, ...2015;

$$
\bar{\Pi}_{P \Phi}-\text { среднее значение целевого инди- }
$$
катора по РФ за наблюдаемый период, с 2005 года.

Коэффициент регрессии $\beta_{\text {РФ, равен }}$ 0,000048, уравнение регрессии, связывающее индикатор Стратегии и макроэкономический показатель, имеет вид: $\Pi_{\text {ВО }}=\Pi_{\text {РФ }}$ х 0,000048 0,33 .

Оценить степень влияния значений индикатора по РФ на значения в регионе позволяет коэффициент $r_{P \Phi}$, определяемый по формуле (9):

$$
r_{P \Phi}=\beta \cdot \frac{\sigma_{P \Phi}}{\sigma_{B O}}=\frac{\operatorname{cov}\left(\Pi_{B O}, \Pi_{P \Phi}\right)}{\sigma_{B O} \cdot \sigma_{P \Phi}} .
$$

где

$$
\sigma_{B O}^{2}=\frac{\sum_{k=0}^{i}\left(\Pi_{k}-\bar{\Pi}\right)^{2}}{n-1}-\text { стандартное отклоне- }
$$

ние целевого индикатора от среднего по региону;

$\bar{\Pi}$ - среднее значение целевого индикатора по региону за наблюдаемый период;

$$
-1 \leq \mathrm{r}_{\text {РФ }} \leq 1 \text {. }
$$

Чем ближе модуль значения коэффициента корреляции к единице, тем теснее корреляционная связь. Коэффициент корреляции равен 0,97 .

Изменение целевого индикатора в процентах оценивается с помощью коэффициента

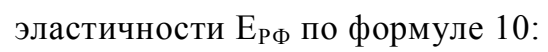

$$
E_{P \Phi}=\beta_{P \Phi} \times \frac{\Pi \Delta}{\Pi_{P \Phi}}=\frac{0,000048 \times 33295}{1,25}=1,27 .
$$

Значение индикатора по региону увеличится на 1,27 \% при увеличении значения индикатора по РФ на один процент.

Оценка внешних рисков недостижения целевого индикатора к 2018 году осуществляется на основе прогноза социальноэкономического развития РФ до 2018 года [6], прогноза научно-технологического развития РФ на долгосрочную перспективу.

Прогнозное значение целевого индикатора в регионе определяется исходя из целевого значения индикатора в 2018 году в прогнозе социально-экономического развития РФ до 2018 года по формуле (11):

$$
\widehat{\Pi}_{2018}=\beta_{P \Phi} \times\left(\Pi_{P \Phi, 2018}-\Pi_{P \Phi}\right)+\Pi=2,5
$$

В сравнении с прогнозным значением целевого индикатора в 2018 году определяется уровень отклонения индикатора от поставленной цели:

a) в отношении целевых индикаторов, большее значение которых отражает лучший результат, по формуле 12:

$$
\Delta \widehat{I}_{2016}=\left(\frac{\widehat{\Pi}_{2016}}{\Pi_{2016}}-1\right) \times 100=\left(\frac{2,5}{3,21}-1\right) \times 100=-22,12<0 .
$$

б) в отношении целевых индикаторов, 


\section{Управление. Моделирование. Информатика}

меньшее значение которых отражает лучший результат, по формуле 13:

$$
\Delta \widehat{I}_{2018}=\left(\frac{\Pi_{2018}}{\widehat{\Pi}_{2018}}-1\right) \times 100 .
$$

В случае если уровень отклонения прогнозного значения индикатора от целевого значения в 2018 году в регионе положителен $\left(\Delta \widehat{I}_{2018}>0\right)$,

то динамика значений индикатора по РФ достаточна для достижения целевого значения индикатора по региону.

В случае если уровень отклонения прогнозного значения индикатора от целевого значения в 2018 году в регионе отрицателен $\left(\Delta \widehat{I}_{2018}\right.$ $<0)$,

то существует риск недостижения стратегических целей, возникает угроза реализации стратегического плана, уровень отклонения прогнозного значения индикатора от целевого значения в 2018 году дает оценку внешних рисков недостижения целевого значения индикатора в 2018 году.

Разница уровней отклонения $\Delta I_{2018}-\Delta \widehat{I}_{2018}$ определяет внутренние риски недостижения целевого значения индикатора в 2018 году, для данного показателя она составляет 0,09\%, что говорит о низких внутренних рисках недостижения (табл. 1).

Аналогичным образом проводится анализ динамики целевых индикаторов в сравнении со значениями для ЦФО.

В качестве макроэкономического показателя, отражающего динамику индикатора Стратегии Воронежской области «Производительность труда в промышленности, млн. руб./чел.», следует рассмотреть аналогичный показатель по
Российской Федерации.

Однако отсутствие статистической информации и целевых значений по этому показателю делает это невозможным.

В качестве показателя макроэкономического развития для РФ выбираем показатель, связанный с рассматриваемым индикатором, такой как «Валовый внутренней продукт».

Данные о значениях показателя «Валовый внутренней продукт» за 2005 - 2015 год по РФ приведены в статистических данных.

Целевой вариант прогноза социальноэкономического развития РФ до 2018 года предусматривает полномасштабную реализацию всех задач, поставленных в указах Президента Российской Федерации от 7 мая 2012 г. N 596606. Прежде всего, это означает выход на сверхвысокую траекторию темпов роста макроэкономических показателей.

В 2018 - 2020 годах среднегодовой рост ВВП должен быть не меньше 6,3\%. Согласно прогнозу в соответствии с целевым вариантом развития рост ВВП в 2018 году по отношению к 2015 году составит $122,04 \%$, целевое значение физического объема ВВП в РФ составит 59649 млрд. руб.

Уровень отклонения прогнозного значения индикатора от целевого значения в 2018 году в регионе отрицателен, существует риск недостижения стратегических целей, есть угроза реализации стратегического плана. Существует высокий внешний риск недостижения целевого значения индикатора в 2018 году [7].

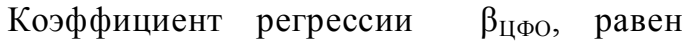
0,00013, уравнение регрессии, связывающее индикатор Стратегии и макроэкономический показатель, имеет вид:

Таблица 1

Шкала рисков недостижения стратегических целей в 2018 году

\begin{tabular}{|l|c|}
\hline $\begin{array}{c}\text { Уровень отклонения прогнозного значения инди- } \\
\text { катора от целевого значения в 2018 году }\end{array}$ & $\begin{array}{c}\text { Степень рисков недостижения стратегических целей в } \\
2018 \text { году }\end{array}$ \\
\hline Положителен & низкая \\
\hline Отрицателен, меньше или равен 5\% & средняя \\
\hline Отрицателен, больше 5\% & высокая \\
\hline
\end{tabular}




\section{Управление. Моделирование. Информатика}

$$
\Pi_{\text {во }}=\Pi_{\text {чфо }} \times 0,00013 .
$$

Коэффициент корреляции равен 0,95 , что говорит о тесной корреляционной связи.

Изменение целевого индикатора в процентах оценивается с помощью коэффициента

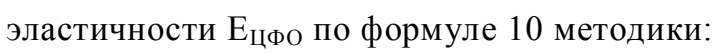

$$
E_{\text {ЦФO }}=\beta_{\text {LФO }} \times \frac{\Pi \Delta}{\Pi_{\text {LФO }}}=0,00013 \times 11934 / 1,25=1,26 .
$$

Значение индикатора по региону увеличится на $1,26 \%$ при увеличении значения индикатора по ЦФО на один процент.

Согласно прогнозу в соответствии с целевым вариантом развития рост ВВП в 2018 году по отношению к 2015 году составит 122,04\%, целевое значение физического объема ВВП в ЦФО составит 21276 млрд. руб. Исходя из этого целевого значения макроэкономического показателя прогнозное значение целевого индикатора в регионе определяется по формуле 11 методики:

$$
\widehat{\Pi}_{L \phi O, 2018}=\beta_{Ц \Phi O} \times\left(\Pi_{L \Phi O, 2018}-\Pi_{L \phi O}\right)+\Pi=2,48
$$

В сравнении с прогнозным значением целевого индикатора в 2018 году определяется уровень отклонения индикатора от поставленной цели по формуле 12 методики:

$$
\Delta \widehat{I}_{2018}=\left(\frac{\widehat{\Pi}_{2018}}{\Pi_{2018}}-1\right) \times 100=\left(\frac{2,48}{3,21}-1\right) \times 100=-22,74<0 .
$$

Динамика по ЦФО ниже, чем по РФ, существует высокий внешний риск недостижения целевого значения индикатора в 2018 году и низкие внутренние риски недостижения [8] .

4. Анализ внутренних и внешних рисков недостижения целевых значений индикатора к 2020 году.

В случае если существует риск недостижения стратегических целей к 2018 году

$$
\left(\Delta \widehat{I}_{2018}<0\right) \text {, }
$$

этот риск сохранится и к 2020 году.

Оценка уровня недостижения целевых значений к 2020 году проводится по формуле (14):

$$
\Delta \widehat{I}_{2020}=\Delta \widehat{I}_{2018} \times 2=22,74 \times 2=-45,48 \%
$$

Уровень отклонения индикатора от поставленной цели к 2020 году также отрицателен, при сохранении динамики целевого индикатора риск недостижения стратегических целей к 2020 году будет возрастать, крайне высок внешний риск недостижения целевого значения индикатора в 2020 году, внутренние риски недостижения целевого значения индикатора в 2020 году незначительны (разница уровней отклонения положительна и составляет 10,8 \%). Разница уровней отклонения $\Delta_{2020}-\Delta \widehat{U}_{2020}$ определяет внутренние риски недостижения целевого значения индикатора в 2020 году.

Анализ рисков недостижения можно провести и на основе Концепции долгосрочного социально-экономического развития Российской Федерации на период до 2020 года. Согласно Распоряжению Правительства РФ от 17 ноября 2008 г. N 1662-р «О Концепции долгосрочного социально-экономического развития Российской Федерации на период до 2020 года» Российская Федерация находится на втором этапе развития (2015 - 2020 годы). На этом этапе намечается рывок в повышении глобальной конкурентоспособности экономики на основе ее перехода на новую технологическую базу (информационные, био- и нанотехнологии), улучшения качества человеческого потенциала и социальной среды, структурной диверсификации экономики. Также планируется достигнуть роста валового внутреннего продукта на 164 - 166 процентов (2020 год к 2015 году), что составит 81132 млрд. руб.

Оценка целевого значения показателя Стратегии с помощью уравнения регрессии составит:

$$
\Pi_{\mathrm{BO}, 2020}=\Pi_{\mathrm{P}, 2020} \quad \times 0,000048-0,33=
$$
3,52 млн. руб./чел.

В сравнении с прогнозным значением целевого индикатора в 2020 году определяется уровень отклонения индикатора от поставленной цели:

$$
\Delta \widehat{I}_{2020}=\left(\frac{\Pi_{2020}}{\Pi_{2020}}-1\right) \times 100=\left(\frac{3,52}{5,24}-1\right) \times 100=-32,82<0
$$

Уровень отклонения прогнозного значения индикатора от целевого значения в 2018 году в регионе отрицателен, существует риск недостижения стратегических целей, есть угроза реализации стратегического плана. Существует высокий внешний риск недостижения целевого 


\section{Управление. Моделирование. Информатика}

значения индикатора в 2020 году (табл. 2).

Выводы:

Предлагаемая методика моделирует внутренние риски недостижения целевого значения индикатора в 2020 году. Среди них следует отметить более высокую по сравнению с ЦФО степень износа основных фондов крупных и средних коммерческих организаций. На конец 2015 года она по региону составляет 44,7\%, в то время как в ЦФО - 39 \%, однако это значение ниже среднего значения по РФ (45,9 \%). Также в регионе ниже уровня по ЦФО и РФ уровень инновационной активности организаций промышленного производства. По итогам 2015 года в Воронежской области он составил 9,8 \%, в то время как в ЦФО - 11,4\%, в РФ - 11,1 \%. В соседних областях значение уровня инновационной активности организаций промышленного производства еще выше: в Курской области $14,7 \%$, в Липецкой области - 18,2 \%. Ниже, чем в ЦФО и РФ и заработная плата на предприятиях промышленности, по данным 2014 года в Воронежской области она составила 22205 руб., в то время как в ЦФО - 26913 руб., в РФ - 32667 руб.

Внешние риски недостижения целевого значения индикатора в 2020 году крайне незначительны и носят преимущественно экономический характер.

Таблица 2

Шкала рисков недостижения стратегических целей в 2020 году

\begin{tabular}{|l|c|}
\hline $\begin{array}{c}\text { Уровень отклонения прогнозного значения инди- } \\
\text { катора от целевого значения в } 2020 \text { году }\end{array}$ & $\begin{array}{c}\text { Степень рисков недостижения стратегических } \\
\text { целей в 2020 году }\end{array}$ \\
\hline Положителен & низкая \\
\hline Отрицателен, меньше или равен $10 \%$ & средняя \\
\hline Отрицателен, больше $10 \%$ & высокая \\
\hline \hline
\end{tabular}

\section{Библиографический список}

1.Афанасьева, Л.В. Методические аспекты оценки показателя интегрального риска в системе стратегического управления регионом [Текст]/ Л.В.Афанасьева, Т.Б.Ткаченко // - Известия ЮЗГУ. Серия: Экономика. Социология. Менеджмент. - 2013. - № 4. -С. 44-49.

2.Кузнецов, Б.Л. Производительность труда как инструмент стратегического развития региона [Текст]/ Б.Л.Кузнецов, Э.К.Мингалеева, Г.Ф.Галиуллина// - Известия Уральского государственного экономического университета. - 2008. - N 2. - С. 97-104.

3.Масыч, М.А. Производительность труда в отраслях промышленности: экономикостатистический анализ [Текст]/ М.А. Масыч, Е.В. Каплюк, А.С. Краснянский, А.В. Тихонина//- Фундаментальные исследования. - 2015. - № 12-3. - С. 605-608.

4.Стрижакова, Е.Н. Производительность труда в промышленности: факторы роста, резервы и модели [Текст] / Е.Н. Стрижакова //Экономика и предпринимательство. - 2014. - № 11-4 (52-4). - С. 493 497.

5.Сухарев, О.С. Структурный анализ развития промышленной системы [Текст]/ О.С. Сухарев, Е.Н. Стрижакова //Национальные интересы: приоритеты и безопасность. - 2014. -№ 41 (278). - С. 26-40.

6.Сухорукова, В.В. Методика выбора приоритетных рынков как объектов развития конкуренции в Воронежской области [Текст]/ В.В. Сухорукова, Д.Ю. Трещевский, О.В. Хорошилова // Русский провинциальный научный журнал «Регион: системы, экономика, управление».- 2016. -№1 (32) - С. 134-140.

7.Ускова, Т.В. Рост производительности труда-стратегическая задача регионов [Текст]/ Т.В. Ускова // Экономические и социальные перемены: факты, тенденции, прогноз. -2008. -№ 4. -C. 15-26.

8. Dyatlov, S. A. Methodology of management innovation hypercompetition [Text] / S. A. Dyatlov, V. M. Vasiltsova, V. S. Vasiltsov, T. L. Bezrukova, B. A. Bezrukov // Asian Social Science. - 2015. - Vol. 11. no. 20. - pp. 165-169.

9. Popkova, E. G. Strategic effectiveness evaluation as integral part of the modern enterprise manage- 
ment [Электронный ресурс] / E.G. Popkova, S.A. Abramov S.A., L.V. Ermolina, E.V. Gandin// Asian Social Science. - 2015. - Vol. 11, № 20. - pp. 16-21.

10. Yakovleva, E.A. Innovation as a Vector of Regional Economic Development and a Necessary Condition for the Progress of the World Economy [Электронный pecypc] / E.A. Yakovleva, N.A. Azarova, E.V. Titova// Asian Social Science. - 2015. - Vol. 11, № 20. - p. 90.

\section{References}

1. Afanas'yeva L.V., Tkachenko T.B. Metodicheskiye aspekty otsenki pokazatelya integral'nogo riska v sisteme strategicheskogo upravleniya regionom [Metodicheskiye aspekty otsenki pokazatelya integral'nogo riska v sisteme strategicheskogo upravleniya regionom]. Izvestiya YUZGU. Seriya: Ekonomika. Sotsiologiya. Menedzhment [Izvestiya YUZGU. Seriya: Ekonomika. Sotsiologiya. Menedzhment]. 2013, no. 4, pp.44-49 (In Russian).

2. Kuznetsov B.L.,.Mingaleyeva E.K., Galiullina G.F. Proizvoditel'nost' truda kak instrument strategicheskogo razvitiya regiona [Proizvoditel'nost' truda kak instrument strategicheskogo razvitiya regiona]. Izvestiya Ural'skogo gosudarstvennogo ekonomicheskogo universiteta [Izvestiya Ural'skogo gosudarstvennogo ekonomicheskogo universiteta]. 2008, no.2, pp.97-104 (In Russian).

3. Masych, M.A. Proizvoditel'nost' truda v otraslyakh promyshlennosti: ekonomiko-statisticheskiy analiz [Proizvoditel'nost' truda $\mathrm{v}$ otraslyakh promyshlennosti: ekonomiko-statisticheskiy analiz]. Fundamental'nyye issledovaniya [Fundamental'nyye issledovaniya]. 2015, no.12-3, pp.605-608 (In Russian).

4. Strizhakova Ye.N. Proizvoditel'nost' truda v promyshlennosti: faktory rosta, rezervy i modeli [Proizvoditel'nost' truda v promyshlennosti: faktory rosta, rezervy i modeli]. Ekonomika i predprinimatel'stvo [Ekonomika i predprinimatel'stvo]. 2014, no. 11-4(52-4), pp. 493-497 (In Russian).

5. Sukharev O.S., Strizhakova Ye.N. Strukturnyy analiz razvitiya promyshlennoy sistemy [Strukturnyy analiz razvitiya promyshlennoy sistemy]. Natsional'nyye interesy: prioritety i bezopasnost' [Natsional'nyye interesy: prioritety i bezopasnost']. 2014, no. 41(278), pp. 26-40 (In Russian).

6. Sukhorukova V.V., Treshchevskiy D.YU., Khoroshilova O.V. Metodika vybora prioritetnykh rynkov kak ob"yektov razvitiya konkurentsii $v$ Voronezhskoy oblasti [Metodika vybora prioritetnykh rynkov kak ob"yektov razvitiya konkurentsii v Voronezhskoy oblasti]. Russkiy provintsial'nyy nauchnyy zhurnal «Region: sistemy, ekonomika, upravleniye» [Russkiy provintsial'nyy nauchnyy zhurnal «Region: sistemy, ekonomika, upravleniye»]. 2016, no. 1 (32), pp. 134-140 (In Russian).

7. Uskova, T.V. Rost proizvoditel'nosti truda-strategicheskaya zadacha regionov [Rost proizvoditel'nosti truda-strategicheskaya zadacha regionov]. Ekonomicheskiye i sotsial'nyye peremeny: fakty, tendentsii, prognoz [Ekonomicheskiye i sotsial'nyye peremeny: fakty, tendentsii, prognoz]. 2008, no. 4, pp.15-26 (In Russian).

8. Vasiltsova V.M., Vasiltsov V.S., Bezrukova T.L., Bezrukov B.A. Methodology of management innovation hypercompetition. Asian Social Science, 2015, Vol. 11, no. 20, pp. 165-169.

9. Popkova, E. G., Abramov S.A., Ermolina L.V., Gandin E.V. Strategic effectiveness evaluation as integral part of the modern enterprise management. Asian Social Science. 2015, Vol. 11, no. 20, pp. 16-21.

10. Yakovleva E.A., Azarova N.A., Titova E.V. Innovation as a Vector of Regional Economic Development and a Necessary Condition for the Progress of the World Economy. Asian Social Science, 2015, Vol. 11, no. 20 , p. 90 .

\section{Сведения об авторах}

Сухорукова Виктория Витальевна - советник отдела сводного прогнозирования регионального развития Департамента экономического развития Воронежской области, кандидат физико-математических наук, доцент, г. Воронеж, Российская Федерация; e-mail: abiti@mail.ru.

Яковлева Елена Александровна - заведующий кафедрой мировой и национальной экономики ФГБОУ ВО «Воронежский государственный лесотехнический университет имени Г.Ф. Морозова», доктор экономических наук, профессор, г. Воронеж, Российская Федерация; e-mail: elena-12-27@mail.ru 
Азарова Наталья Анатольевна - доцент кафедры мировой и национальной экономики ФГБОУ ВО «Воронежский государственный лесотехнический университет имени Г.Ф. Морозова», кандидат экономических наук, г. Воронеж, Российская Федерация; e-mail: azarovarsd@rambler.ru

\section{Information about authors}

Sukhorukova Victoria Vitalyevna - Advisor of the Department for forecasting regional development, Economic development Department of Voronezh region, $\mathrm{PhD}$ in Physics and Mathematical Sciences, Associate Professor, Voronezh, Russian Federation; e-mail: abiti@mail.ru.

Yakovleva Elena Alexandrovna - Head of «World and National Economy» department, Federal State Budget Education Institution of Higher Education «Voronezh State University of Forestry and Technologies named after G.F. Morozov», DSc in Economics, Professor, Voronezh, Russian Federation, e-mail: elena-1227@mail.ru.

Azarova Natalia Anatolievna - Associate Professor «World and National Economy» department, Federal State Budget Education Institution of Higher Education «Voronezh State University of Forestry and Technologies named after G.F. Morozov», PhD in Economics, Voronezh, Russian Federation; e-mail: azarovarsd@rambler.ru. 